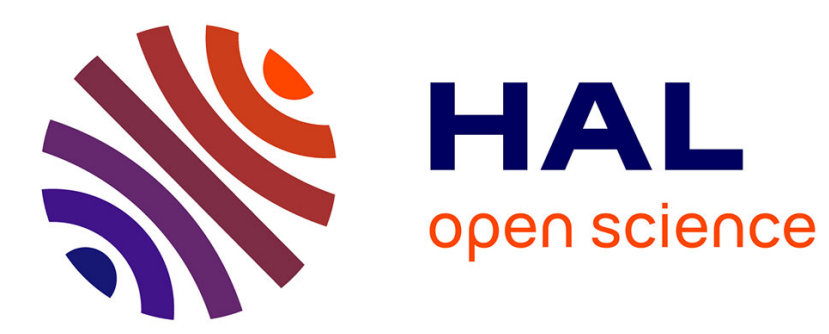

\title{
Fatigue symptoms relate to systemic inflammation in patients with type 2 diabetes
}

Julie Lasselin, Sophie Layé, Sandra Dexpert, Agnès Aubert, Concepción González, Henri Gin, Lucile Capuron

\section{- To cite this version:}

Julie Lasselin, Sophie Layé, Sandra Dexpert, Agnès Aubert, Concepción González, et al.. Fatigue symptoms relate to systemic inflammation in patients with type 2 diabetes. Brain, Behavior, and Immunity, 2012, 26 (8), pp.1211-1219. 10.1016/j.bbi.2012.03.003 . hal-02646383

\section{HAL Id: hal-02646383 \\ https://hal.inrae.fr/hal-02646383}

Submitted on 29 May 2020

HAL is a multi-disciplinary open access archive for the deposit and dissemination of scientific research documents, whether they are published or not. The documents may come from teaching and research institutions in France or abroad, or from public or private research centers.
L'archive ouverte pluridisciplinaire HAL, est destinée au dépôt et à la diffusion de documents scientifiques de niveau recherche, publiés ou non, émanant des établissements d'enseignement et de recherche français ou étrangers, des laboratoires publics ou privés. 


\title{
Fatigue symptoms relate to systemic inflammation in patients with type 2 diabetes
}

\author{
Julie Lasselin $^{\mathrm{a}, \mathrm{b}}$, Sophie Layé ${ }^{\mathrm{a}, \mathrm{b}}$, Sandra Dexpert ${ }^{\mathrm{a}, \mathrm{b}}$, Agnès Aubert ${ }^{\mathrm{a}, \mathrm{b}}$, Concepcion Gonzalez ${ }^{\mathrm{c}}$, Henri Gin ${ }^{\mathrm{c}}$, \\ Lucile Capuron $^{\mathrm{a}, \mathrm{b}, *}$
}

a INRA, Laboratory of Nutrition and Integrative Neurobiology (NutriNeuro), UMR 1286, F-33076 Bordeaux, France
b Univ. Bordeaux, Laboratory of Nutrition and Integrative Neurobiology (NutriNeuro), UMR 1286, F-33076 Bordeaux, France
${ }^{\mathrm{c}}$ CHU Bordeaux, Hôpital du Haut-Lévêque, Service Nutrition-Diabétologie, F-33604 Pessac, France

\section{A R T I C L E I N F O}

\section{Article history:}

Received 2 January 2012

Received in revised form 9 March 2012

Accepted 9 March 2012

Available online 25 March 2012

\section{Keywords:}

Fatigue

Dimensions

Diabetes

Types 1 and 2

Inflammation

Cytokines

$C$ reactive protein

Interleukin-6

Neopterin

Adiposity

\begin{abstract}
A B S T R A C T
Fatigue is frequent in patients with diabetes and this symptom appears to be more prominent in type 2 rather than type 1 diabetic subjects. Chronic inflammation represents one characteristic of type 2 diabetes that may contribute to fatigue symptoms. This possibility was assessed in a sample of 20 type 2 diabetic patients relatively to a group of 20 type 1 diabetic subjects. Specific dimensions of fatigue, including general fatigue, physical fatigue, reduced activity, mental fatigue and reduced motivation, were assessed using the Multidimensional-Fatigue-Inventory (MFI). Biological assays comprised the measurement of serum inflammatory markers [high-sensitive C-reactive-protein (hSCRP), high-sensitive interleukin-6 (hsIL-6), high-sensitive tumor-necrosis-factor- $\alpha$ (hsTNF- $\alpha$ ) and neopterin]. Clinical parameters including indexes of adiposity were collected. In comparison to type 1 diabetic subjects, patients with type 2 diabetes exhibited higher fatigue scores, notably in the dimensions of general fatigue, physical fatigue and reduced activity, together with greater levels of inflammatory markers that correlated with indexes of adiposity. Regression analyses controlling for diabetes duration, insulin treatment status, glycemic control and fasting status, indicated that levels of inflammatory markers, in particular hsIL-6, hsCRP and neopterin, were associated with MFI fatigue dimensions in type 2 diabetic patients. Mediation analyses revealed that adiposity did not significantly account for the relationship of inflammatory markers with fatigue scores albeit coefficient regressions decreased somewhat when this variable was controlled for in regression models. These findings indicate that systemic low-grade inflammation relates to fatigue symptoms in patients with type 2 diabetes and suggest the involvement of inflammatory processes in the pathophysiology of diabetes-related fatigue.
\end{abstract}

(c) 2012 Elsevier Inc. All rights reserved.

\section{Introduction}

Fatigue represents a common complaint of patients with diabetes as it can be reported in up to $60 \%$ of patients (Drivsholm et al., 2005; Fritschi and Quinn, 2010). This symptom not only impacts the patient's quality of life and compliance to treatment, but it is also associated with an increased risk of disease complications (Fritschi and Quinn, 2010). In a recent comparative study assessing fatigue symptoms in patients with type 1 and type 2 diabetes, we found that fatigue was particularly prominent in patients with type 2 diabetes and concerned primarily physical aspects rather than mental components. In patients with type 1 diabetes, fatigue scores were comparable to scores measured in healthy volunteers (Lasselin et al., 2012).

\footnotetext{
* Corresponding author at: INRA 1286, Laboratory of Nutrition and Integrative Neurobiology (NutriNeuro), University Victor Segalen Bordeaux 2, 146 rue Léo Saignat, F-33076 Bordeaux, France. Tel.: +33 557571233; fax: +33 557571227.

E-mail address: lucile.capuron@bordeaux.inra.fr (L. Capuron).
}

The pathophysiological mechanisms leading to the development of fatigue symptoms in patients with type 2 diabetes remain largely unknown. While many biological and psychological factors may contribute, several lines of evidence point to the possibility that inflammatory factors are also involved. In support of this notion, recent data indicate that blockade of the inflammatory cytokine interleukin (IL)-1 $\beta$ with the monoclonal anti-IL1 $\beta$ antibody, XOMA052, partly improves motor fatigue in patients with type 2 diabetes (Cavelti-Weder et al., 2011). The ability of pro-inflammatory cytokines (e.g., IL-6, IL-1 $\beta$, tumor necrosis factor (TNF)- $\alpha$ ) to act on the brain and to induce behavioral symptoms, including fatigue, is well documented in both experimental and clinical studies (Capuron and Miller, 2011; Dantzer et al., 2008; Kelley et al., 2003; Majer et al., 2008). At the clinical level, the involvement of inflammation in the development of fatigue symptoms has been shown in patients with chronic medical conditions, including cancers, multiple sclerosis and chronic fatigue syndrome (Bower et al., 2002; Flachenecker et al., 2004; Fletcher et al., 2009; Heesen et al., 2006; Orre et al., 2009; Raison et al., 2009). Moreover, studies conducted in our group have shown that inflammation plays a 
major role in the development of fatigue symptoms in medically ill patients treated with cytokines, notably interferon- $\alpha$ (Capuron et al., 2002, 2007). Similar results were found in the elderly subject (Capuron et al., 2011a). All of these conditions share in common the chronic activation of immune/inflammatory processes. Similar to these conditions, diabetes is characterized by a chronic, lowgrade, inflammatory state. While this activation of inflammatory processes has been described in both type 1 and type 2 phenotypes (Basu et al., 2005; Devaraj et al., 2006; Pickup et al., 2000; Su et al., 2011), increased levels of circulating inflammatory markers were found to be significantly more prominent in type 2 diabetes (Alexandraki et al., 2008; Pham et al., 2011). Whereas increased inflammatory markers, notably C-Reactive Protein (CRP), were found to relate to long disease duration and diabetes complications in type 1 diabetes (Devaraj et al., 2007; Treszl et al., 2004), systemic inflammation in type 2 diabetes is believed to originate, at least partially, from the adipose tissue (Mraz et al., 2011; Pedersen et al., 2003; Sam et al., 2009). Adiposity is a distinguishing characteristic of type 2 diabetes that may easily promote the development of chronic inflammation, as adipocytes and infiltrated macrophages in the adipose tissue have the ability to secrete pro-inflammatory factors (Trayhurn and Wood, 2004; Wellen and Hotamisligil, 2003). Interestingly, systemic inflammation was found in many studies to be associated with an increased risk of developing type 2 diabetes, suggesting that inflammatory processes may also contribute to the pathophysiology of the disease (Duncan et al., 2003; Festa et al., 2002; Hu et al., 2004; Schmidt et al., 1999; Spranger et al., 2003). Given the well documented role of inflammation in the development of fatigue in chronic or immune-based conditions (Bower, 2007; Capuron and Miller, 2011; Miller et al., 2008), it appears highly possible that systemic inflammation also contributes to fatigue symptoms in patients afflicted with diabetes, notably of type 2 phenotype.

The objective of this study was to assess the inflammatory status of patients with type 2 diabetes comparatively to a group of type 1 diabetic subjects and to evaluate the involvement of systemic low-grade inflammation in fatigue symptoms in the same population.

\section{Patients and methods}

\subsection{Participants}

This study included 20 patients with type 2 diabetes and 20 patients with type 1 diabetes recruited from the service of diabetology at the Haut-Lévêque Hospital in Bordeaux, France. Type 2 diabetic patients had a personal history of overweight, 10 of them were treated with insulin and the remainder 10 patients were either treated with oral antidiabetic medications $(N=6)$ or untreated $(N=4)$. All type 1 diabetic patients were treated with insulin, they had no personal history of overweight and they had shown significant weight loss at the onset of the disease. Clinical variables including the medical history of patients, duration of diabetes, glycated hemoglobin (HbA1C) levels (as indexes of diabetes control), treatment status, body mass index (BMI) and waist circumference (as indexes of adiposity) were collected in all participants. Patients with current major depressive disorder, as assessed by a structured clinical diagnostic interview with the Mini-International-Neuropsychiatric-Interview (MINI) (Sheehan et al., 1998), patients treated with antidepressants or neuroleptics, and patients suffering from chronic inflammatory medical conditions were excluded.

The study was approved by the local Committee for the Protection of Persons (CPP Bordeaux). All participants were adults and written informed consent was obtained from each of them after reading a complete description of the study.

\subsection{Biological measurements}

Blood samples were obtained for the measurement of serum concentrations of the inflammatory markers, high sensitivity (hs)CRP, hsIL-6 and hsTNF- $\alpha$, as well as neopterin as marker of macrophage activation. Samples were centrifuged (10 min, $1000 \mathrm{~g}, 4^{\circ} \mathrm{C}$ ) after clotting and sera were stored at $-80^{\circ} \mathrm{C}$ until the assays. Biological markers were assayed by enzyme-linked immunosorbent assay (ELISA) according to the manufacturer's specifications (hsIL-6 and hsTNF- $\alpha$ : R\&D Systems, Minneapolis, Minnesota; hsCRP: CYT298, Millipore, Billerica, Massachusetts; neopterin: IBL International, Hamburg, Germany). Intra- and inter-assay variability were respectively $\pm 7.4 \%$ and $\pm 7.8 \%$ for hsIL-6, $\pm 5.3 \%$ and $\pm 8.4 \%$ for hsTNF- $\alpha, \pm 4.6 \%$ and $\pm 6.0 \%$ for hsCRP, 3.6 $6.8 \%$ and $7.6-10.3 \%$ for neopterin. Sensitivities were $0.039 \mathrm{pg} / \mathrm{mL}$ for hsIL-6, $0.106 \mathrm{pg} / \mathrm{mL}$ for hsTNF- $\alpha, 0.20 \mathrm{ng} / \mathrm{mL}$ for hsCRP and $0.7 \mathrm{nmol} / \mathrm{L}$ for neopterin.

\subsection{Assessment of fatigue}

Fatigue was assessed using the Multidimensional Fatigue Inventory (MFI), a 20-item self-report questionnaire measuring five dimensions of fatigue corresponding respectively to general fatigue, physical fatigue, mental fatigue, reduced activity, and reduced motivation (Smets et al., 1995). Each dimension contains four items, scored from 1 to 5 with higher scores corresponding to greater levels of fatigue.

\subsection{Data analyses and statistics}

Raw values for the biological markers, hsIL-6, hsCRP and hsTNF$\alpha$, were log-transformed because of non-normality. Characteristics of participants were compared across diabetes phenotype (type 1 versus type 2 diabetes) using two-sample $t$-tests for continuous variables and Chi-square tests for categorical variables. MFI fatigue scores were compared across groups using analysis of covariance (ANCOVA) controlling for insulin treatment status, diabetes duration and HbA1C levels. Due to logistic and/or medical constraints inherent to clinical setting and to the situation of diabetes, the collection of blood samples in fasting conditions was possible only in $30 \%$ of study participants. In order to control for this variation across subjects, analyses performed on biological markers were therefore systemically adjusted for fasting status in addition to insulin treatment status, diabetes duration and HbA1C levels. Three participants exhibited extreme (outlier) values (>3 SD above the mean) for at least one inflammatory marker and thus were excluded from analyses performed on biological data. Factor (principal component) analysis with varimax rotations was performed on biological markers to assess their interconnections and to eventually extract one composite inflammatory component. The relationship of adiposity (i.e., BMI and waist circumference) with inflammatory markers was estimated using Bravais-Pearson correlations performed in the whole population under study and separately in each diabetes phenotype. Multivariate regression analyses entering biological markers as predictors in separate models were performed to assess the relationship of levels of inflammatory markers with fatigue symptom dimensions in each diabetes phenotype. Models were adjusted for diabetes duration, HbA1C levels, insulin treatment and fasting status. Contribution of adiposity to the relationship between systemic inflammation and fatigue symptoms was assessed using the method recommended by Baron and Kenny (1986). According to this method, mediation takes place when (1) the initial variable is significantly associated with the outcome; (2) the initial variable is significantly correlated with the mediator; (3) the mediator has a significant effect on the outcome, when controlling for the initial variable; and 
(4) the relationship between the initial variable and the outcome is reduced or no longer significant, when controlling for the mediator. The significance of the mediation (indirect effect) was tested using both conservative (Sobel) and non-parametric tests (bootstrapping resampling method as described by Preacher and Hayes, 2008). Statistical analyses were performed with SPSS Statistics version 19. All probabilities were two-sided with the degree of significance set at $p<0.05$.

\section{Results}

\subsection{Characteristics of participants}

As shown in Table 1, there was no significant difference between type 1 versus type 2 diabetic patients in terms of age and gender. Duration of diabetes (time since diagnosis) was longer in type 1 diabetic patients compared to type 2 diabetic subjects. Moreover, HbA1C levels were lower (indicative of a better control of diabetes) in type 1 diabetic patients. Finally, adiposity markers (i.e., BMI, waist circumference) were higher in type 2 diabetic patients compared to type 1 diabetic subjects.

\subsection{Inflammatory markers}

Overall, levels of circulating inflammatory markers were higher in type 2 diabetic patients compared to type 1 diabetic subjects (Fig. 1). More specifically, patients with type 2 diabetes exhibited significantly higher concentrations of hsCRP, hsTNF- $\alpha$ and neopterin (respectively, $F(1,31)=9.8, p<.01 ; F(1,31)=5.1, p<.05$ and $F(1,31)=4.4, p<.05)$. Similarly, concentrations of hsIL-6 were higher in type 2 diabetic patients compared to type 1 diabetic subjects, but at a statistical trend level only $(F(1,31)=3.6, p=.07)$. Factor analysis performed on inflammatory markers in the whole population under study revealed that these markers were interconnected and composed altogether one composite inflammatory dimension explaining $49 \%$ of the variance.

As expected, BMI and waist circumference correlated significantly with the composite measure of inflammation and with levels of individual inflammatory markers in the whole population under study, with increased adiposity indexes being related to higher levels of inflammation (Table 2 and Fig. 2). Further correlational analyses performed separately in each of the two diabetic populations revealed that these correlations were primarily significant in type 2 diabetic patients (Table 2).

Given the association of BMI and waist circumference with inflammatory markers in type 2 diabetic patients, complementary analyses using multivariate regression models were performed to assess the contribution of these variables to the relationship of

Table 1

Characteristics of study participants.

\begin{tabular}{lll}
\hline & Type 1 diabetes & Type 2 diabetes \\
\hline Sample size $(N)$ & 20 & 20 \\
Age, mean (SD) & $51.8(14.1)$ & $50.3(13.5)$ \\
Women, $n(\%)$ & $5(25)$ & $3(15)$ \\
Time since diabetes diagnosis, years (SD) & $17.8(13.9)$ & $9(6.7)^{*}$ \\
HbA1C, \% (SD) & $7.5(1.8)$ & $9.7(2.4)^{* *}$ \\
BMI, kg/m $(\mathrm{SD})$ & $25.8(4.2)$ & $31.4(5.2)^{* * *}$ \\
Waist circumference, cm $(\mathrm{SD})^{\mathrm{a}}$ & $91.8(12.0)$ & $108.6(13.3)^{* * * *}$ \\
\hline
\end{tabular}

Analyses were performed using two-sample $t$-tests for continuous variables and chi-square tests for categorical variables.

Abbreviations: BMI: body mass index; HbA1C: glycated hemoglobin.

$p<.05$ versus type 1 diabetic patients.

** $p<.01$ versus type 1 diabetic patients.

*** $p<.001$ versus type 1 diabetic patients.

a Waist circumference value was missing in three type 2 diabetic patients. type 2 diabetes with systemic inflammation (Table 3). Consistent with results presented in Fig. 1, type 2 diabetes was significantly associated with levels of inflammatory markers. This association did not remain significant when controlling for BMI or waist circumference, suggesting that adiposity was one major determinant of systemic inflammation in type 2 diabetes.

\subsection{Fatigue symptoms and association with adiposity}

Compared to type 1 diabetic patients, patients with type 2 diabetes exhibited higher MFI total scores $(F(1,35)=8.8, p<.01)$, particularly in the dimensions of general fatigue, physical fatigue and reduced activity $(F(1,35)=12.7, p<.01, F(1,35)=12.2, p<.01$ and $F(1,35)=7.6, p<.01$, respectively) (Table 4 ), consistent with previous findings (Lasselin et al., 2012). Scores of mental fatigue and reduced motivation were comparable across the two diabetic populations $(F(1,35)=2.2, \quad p=.15$ and $F(1,35)=.55, p=.46$, respectively).

No significant relationship was found between fatigue symptoms and BMI or waist circumference in patients with type 1 diabetes. In type 2 diabetic patients, however, BMI was associated with the specific dimensions of mental fatigue and decreased motivation ( $\beta=.770, p<.05$ and $\beta=.768, p<.05$, respectively). No significant association was found with the dimensions of general fatigue, physical fatigue and reduced activity. Similarly, no significant relationship was found between waist circumference and fatigue symptoms in type 2 diabetic patients, except with the dimension of mental fatigue at a marginal statistical level only $(\beta=.715, p=.05)$. Mediation analyses revealed that systemic inflammation (hsIL6, hsCRP and composite inflammation in particular) significantly accounted for the associations of BMI with scores of mental fatigue and reduced motivation, as these associations did not remain significant when controlling for inflammatory markers (e.g., $\beta=.382, p=.33$ for mental fatigue and $\beta=.290$, $p=.45$ for reduced motivation, with control of composite inflammation). The largest mediating effect was obtained with hsIL6 (Sobel: $Z=1.85, p=.06$ for mental fatigue and $Z=2.21, p<.05$ for reduced motivation). Similar results were obtained with the nonparametric bootstrapping resampling method (result not shown).

\subsection{Relationship between inflammation and fatigue symptom dimensions}

Separate multivariate regression analyses controlling for diabetes duration, insulin treatment, $\mathrm{HbA} 1 \mathrm{C}$ levels and fasting status were conducted to assess the relationship of inflammatory markers with fatigue scores. While no significant relationship was found in patients with type 1 diabetes, analyses revealed significant associations between inflammatory markers and fatigue symptom dimensions in patients with type 2 diabetes. In those patients, the composite measure of inflammation and the specific inflammatory markers of hsIL- 6 and hsCRP were associated with MFI total fatigue scores, notably with the dimensions of mental fatigue and reduced motivation, with higher levels of inflammation predicting greater fatigue scores. HsCRP and hsIL-6 were also positively associated with the dimension of reduced activity, but at a statistical trend level only. A significant association was also found between neopterin and scores of general fatigue in type 2 diabetic patients, with higher neopterin levels predicting more intense general fatigue in these patients (Table 5).

Given the significant associations found between adiposity (BMI in particular) with both inflammation and fatigue symptoms, mediation analyses were conducted to test the indirect effect of adiposity on the relationship of inflammatory markers with fatigue scores in type 2 diabetic patients. Although some of the regression coefficients decreased somewhat when controlling for BMI in 

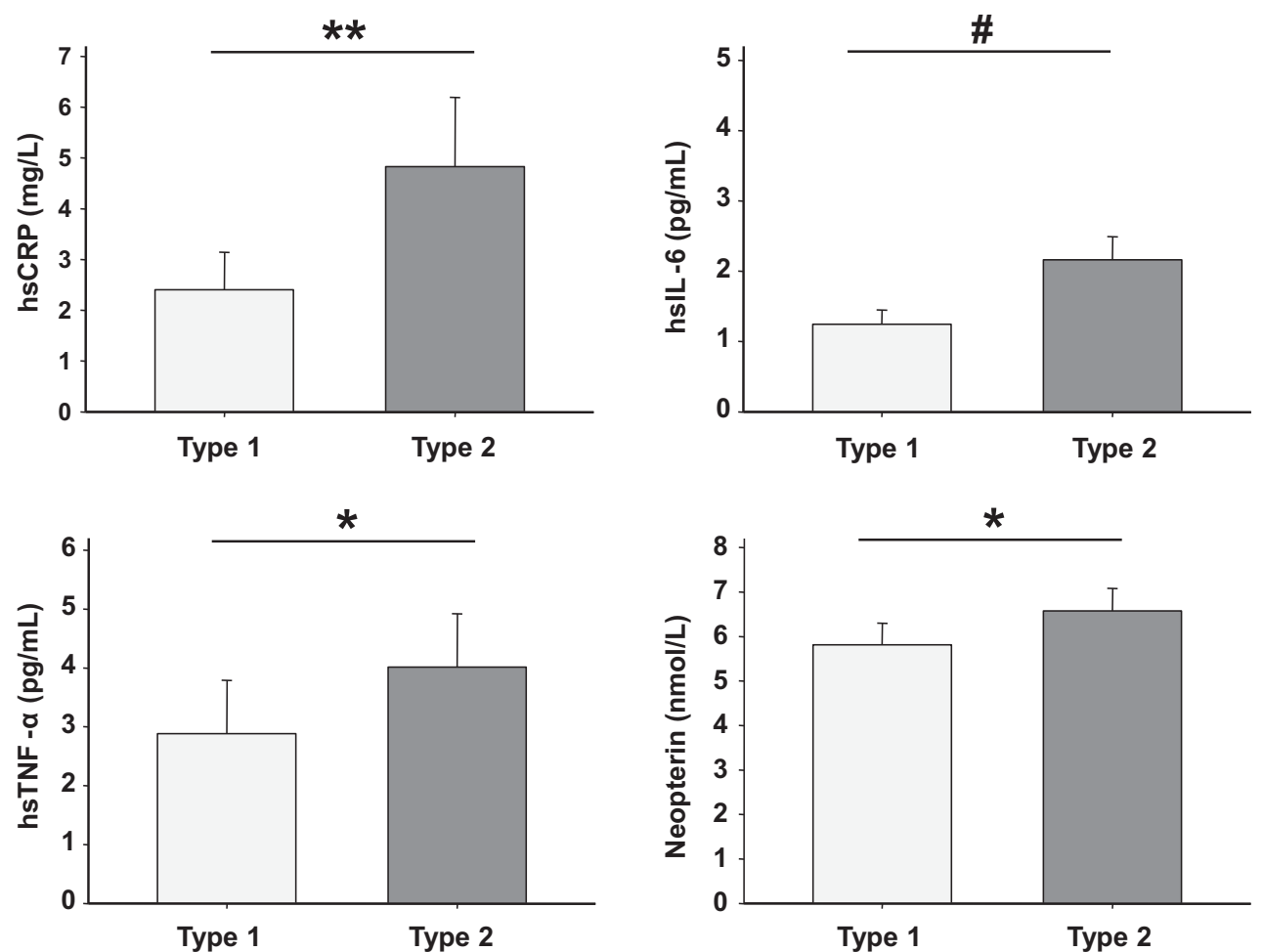

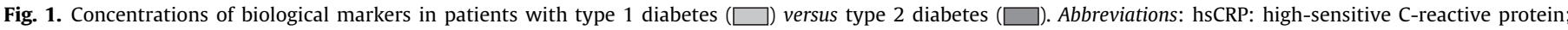

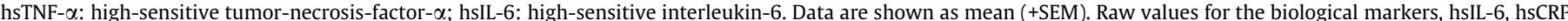

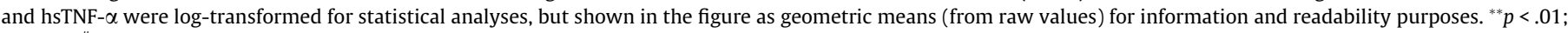
${ }^{*} p<.05 ;{ }^{*} p=.07$.

Table 2

Relationship of adiposity (e.g., body mass index and waist circumference) with inflammatory markers.

\begin{tabular}{|c|c|c|c|c|c|c|}
\hline & \multicolumn{2}{|c|}{ All participants } & \multicolumn{2}{|c|}{ Type 1 diabetes } & \multicolumn{2}{|c|}{ Type 2 diabetes } \\
\hline & BMI & Waist circumference & BMI & Waist circumference & BMI & Waist circumference \\
\hline hsCRP & $.494^{* *}$ & $.480^{* *}$ & .067 & .115 & $.747^{* * * *}$ & $.712^{* *}$ \\
\hline hsIL-6 & $.445^{* *}$ & $.470^{* *}$ & .163 & .242 & $.528^{*}$ & $.446^{\#}$ \\
\hline hsTNF- $\alpha$ & $.421^{* *}$ & $.424^{* *}$ & .369 & .292 & .232 & .312 \\
\hline Neopterin & .205 & $.331^{*}$ & .073 & .194 & .193 & .334 \\
\hline Composite inflammation & $.569^{* * * *}$ & $.611^{* * * *}$ & .257 & .323 & $.656^{* *}$ & $.688^{* *}$ \\
\hline
\end{tabular}

Bravais-Pearson correlations $(R)$.

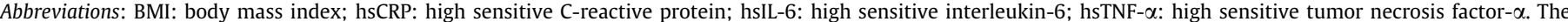

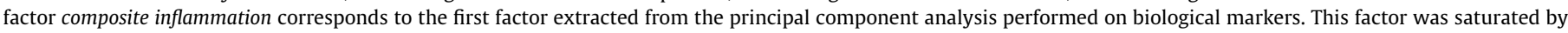
each biological marker (e.g., hsCPR, hsIL-6, hsTNF- $\alpha$ and neopterin) and explained $49 \%$ of the variance.

${ }^{*} p<.05$.

** $p<.01$.

*** $p<.001$.

\# $p=.08$.

multiple regression models (Table 5), mediation analyses revealed that, overall, this variable did not significantly mediate the relationship of systemic inflammation with fatigue symptoms in type 2 diabetic patients (Sobel; all $p>.05$ ), supporting the independent and direct effect of inflammatory markers on fatigue scores. Similar results were obtained with respect to waist circumference and when using non-parametric bootstrapping resampling method (results not shown).

\section{Discussion}

Results from the present study indicate a higher prevalence of fatigue symptoms together with increased signs of systemic inflammation in patients with type 2 diabetes relatively to type 1 diabetic subjects. Consistent with our previous data (Lasselin et al., 2012), fatigue symptoms in type 2 diabetic patients manifested primarily in the dimensions of general fatigue, physical fatigue and reduced activity. Interestingly, significant associations were found between fatigue symptom dimensions and circulating levels of inflammatory markers in type 2 diabetic patients, suggesting the involvement of inflammatory processes in the development of type 2 diabetes-related fatigue. To our knowledge, this is the first time that such an association is shown in a diabetic population.

In line with previous reports (Alexandraki et al., 2008; Pham et al., 2011), low grade inflammation was found to be more prominent in type 2 diabetic patients compared to type 1 diabetic 

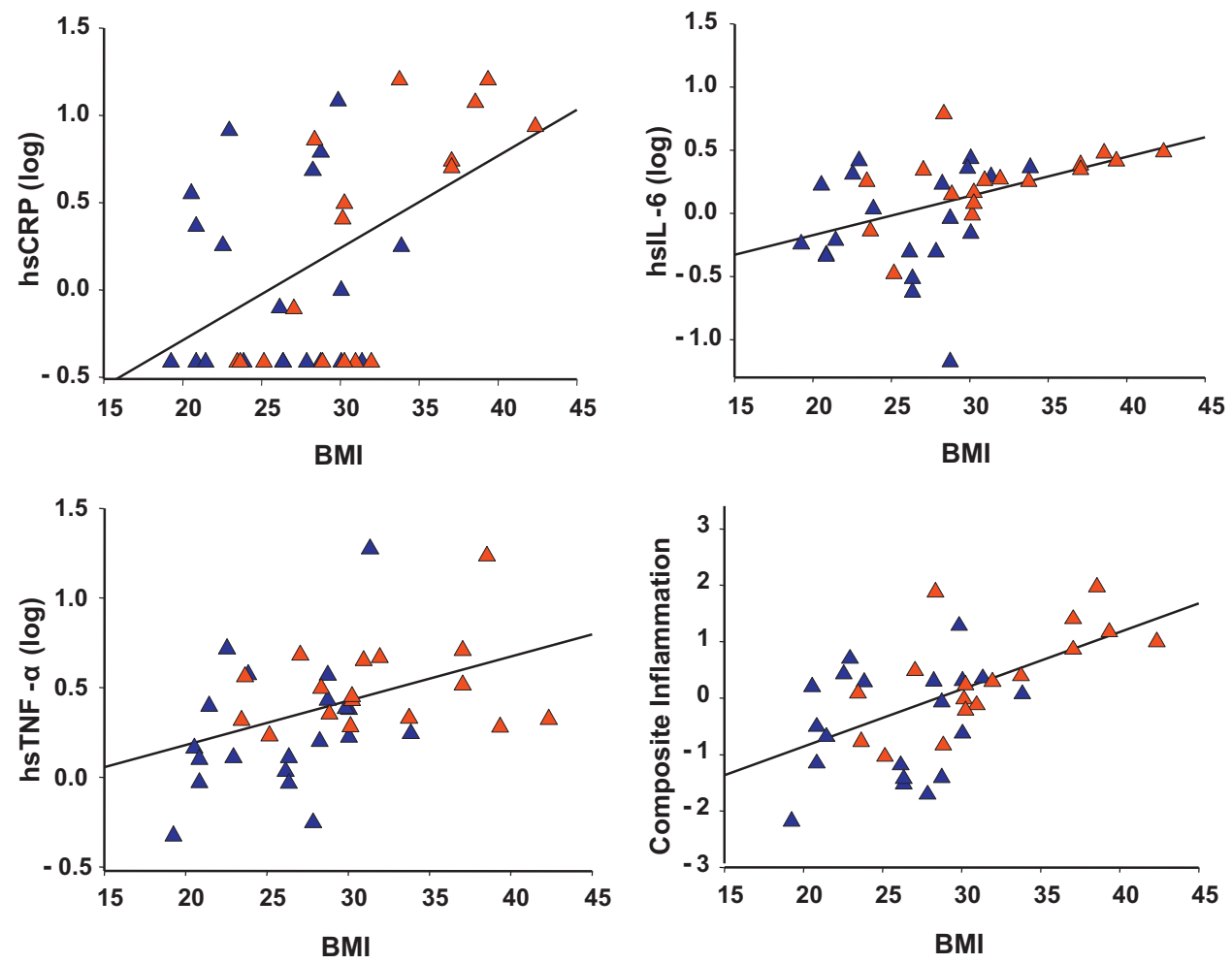

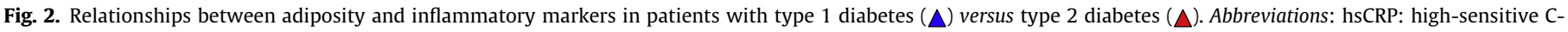

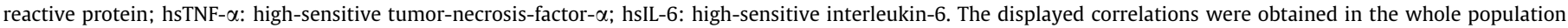
under study. Values for hsCRP, hsIL-6 and hsTNF- $\alpha$ were log-transformed for statistical analyses.

Table 3

Influence of adiposity on the relationship between type 2 diabetes and inflammatory markers.

\begin{tabular}{|c|c|c|c|c|c|c|c|c|c|c|c|c|c|c|c|}
\hline \multirow[t]{3}{*}{ Predictors } & \multicolumn{15}{|c|}{ Inflammatory markers } \\
\hline & \multicolumn{3}{|l|}{ hsCRP } & \multicolumn{3}{|l|}{ hsIL6 } & \multicolumn{3}{|c|}{ hsTNF- $\alpha$} & \multicolumn{3}{|c|}{ Neopterin } & \multicolumn{3}{|c|}{ Composite inflammation } \\
\hline & $\beta$ & $F$ & $p$ & $\beta$ & $F$ & $p$ & $\beta$ & $F$ & $p$ & $\beta$ & $F$ & $p$ & $\beta$ & $F$ & $p$ \\
\hline \multicolumn{16}{|l|}{ Initial model } \\
\hline Type 2 diabetes & .561 & 9.8 & $<.01$ & .381 & 3.6 & .07 & .427 & 5.0 & $<.05$ & .368 & 4.4 & $<.05$ & .616 & 10.8 & $<.01$ \\
\hline BMI & .495 & 12.3 & $<.01$ & .470 & 9.8 & $<.01$ & .458 & 10.0 & $<.01$ & .276 & 3.7 & .06 & .612 & 19.6 & $<.001$ \\
\hline Waist circumference & .474 & 10.5 & $<.01$ & .463 & 9.7 & $<.01$ & .439 & 8.8 & $<.01$ & .342 & 5.9 & $<.05$ & .614 & 19.8 & $<.001$ \\
\hline $\begin{array}{l}\text { Model controlling for } B \Lambda \\
\text { Type } 2 \text { diabetes }\end{array}$ & .282 & 1.5 & .23 & .001 & .01 & .99 & .099 & .2 & .68 & .255 & 1.2 & .29 & .211 & .9 & .36 \\
\hline $\begin{array}{l}\text { Model controlling for } w \\
\text { Type } 2 \text { diabetes }\end{array}$ & .304 & 1.6 & .21 & -.020 & .01 & .94 & .115 & .2 & .64 & .150 & .4 & .53 & .184 & .6 & .43 \\
\hline \multicolumn{16}{|c|}{ Separate effect of covariates } \\
\hline - Diabetes duration & -.085 & .2 & .62 & -.255 & 2.4 & .13 & .131 & 6 & .44 & .294 & 3.0 & .08 & -.018 & .01 & .92 \\
\hline - HbA1C levels & -.185 & 1.2 & .27 & .150 & .8 & .38 & -.030 & .03 & .86 & -.394 & 6.4 & $<.05$ & -.128 & 6 & .45 \\
\hline - Insulin treatment & .318 & 3.9 & .06 & -.250 & 2.3 & .14 & -.243 & 2.2 & .15 & -.057 & .1 & .74 & -.079 & .2 & .64 \\
\hline - Fasting status & .243 & 2.2 & .15 & -.238 & 2.1 & .16 & -.077 & .2 & .65 & .061 & .1 & .72 & -.017 & .01 & .92 \\
\hline
\end{tabular}

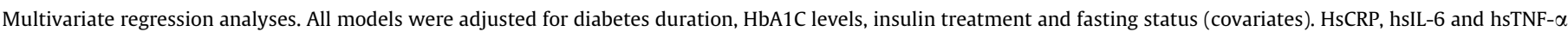

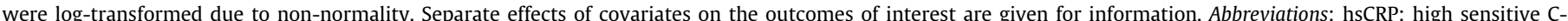

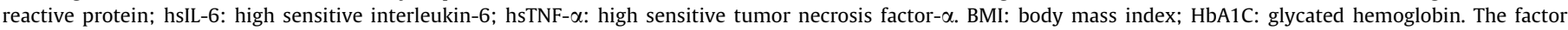

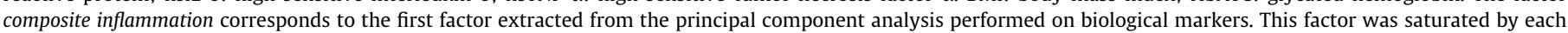
biological marker (e.g., hsCPR, hsIL-6, hsTNF- $\alpha$ and neopterin) and explained $49 \%$ of the variance.

subjects. In patients with type 2 diabetes, systemic inflammation was characterized relatively to type 1 diabetic subjects by increased circulating concentrations of hsCRP, hsIL- 6 and hsTNF- $\alpha$ together with increased levels of neopterin, indicative of macrophage activation. These biological variations were interconnected suggesting the activation of common inflammatory pathways.
The finding that neopterin was higher in type 2 diabetic patients comparatively to type 1 diabetic subjects is in accordance with recent data showing greater concentrations of this markers in a larger group of type 2 diabetic patients in comparison to healthy subjects (Baris et al., 2009). Interestingly, systemic inflammation in patients with type 2 diabetes correlated with adiposity markers 
Table 4

Fatigue symptoms in type 1 versus type 2 diabetes.

\begin{tabular}{lll}
\hline & Type 1 diabetes & Type 2 diabetes \\
\hline MFI total score, mean (SD) & $43.8(13.7)$ & $52.6(11.3)^{* *}$ \\
Fatigue symptom dimensions & & \\
General fatigue, mean (SD) & $9.7(3.6)$ & $12.4(2.9)^{* *}$ \\
Physical fatigue, mean (SD) & $9.2(2.9)$ & $12.0(3.0)^{* *}$ \\
Reduced activity, mean (SD) & $8.5(3.4)$ & $11.0(2.8)^{* *}$ \\
Mental fatigue, mean (SD) & $8.4(3.2)$ & $8.7(3.0)$ \\
Reduced motivation, mean (SD) & $8.1(3.4)$ & $8.6(2.7)$ \\
\hline
\end{tabular}

ANCOVA controlling for insulin treatment status, diabetes duration and HbA1C levels.

Fatigue symptom dimensions were assessed using the Multidimensional Fatigue Inventory (MFI).

$p<.01$ versus type 1 diabetic patients.

(e.g., BMI and waist circumference), with increased adiposity associating with higher inflammation. Moreover, the relationship of type 2 diabetes with systemic inflammation was significantly reduced when controlling for adiposity markers (i.e., BMI or waist circumference) suggesting that adiposity was a major determinant of type-2 diabetes related inflammation. This result supports the notion that low-grade systemic inflammation in type 2 diabetes originates, at least partially, from the adipose tissue and is consistent with previous reports documenting associations between circulating levels of inflammatory markers and abdominal fat mass in cohorts of type 2 diabetic subjects and in the elderly afflicted with type 2 diabetes (Pedersen et al., 2003; Sam et al., 2009).

The finding that systemic low-grade inflammation was associated with fatigue symptom dimensions in patients with type 2 diabetes is particularly original, and suggests the involvement of inflammatory processes in the pathophysiology of diabetes-related fatigue. This result is in accordance with the well documented role of inflammation in the development of behavioral alterations, including fatigue (Capuron and Miller, 2011; Dantzer et al., 2008; Kelley et al., 2003; Majer et al., 2008). The association of inflammatory markers with fatigue symptoms was found in multivariate regression models controlling for diabetes duration, insulin treatment status and HbA1C levels, attesting of the strength of the relationship and indicating that it did not rely on confounding factors nor it was explained by variations in diabetes length, treatment options and/or glycemic control. Moreover, mediation analyses revealed that adiposity did not significantly account for the relationship of inflammatory markers with fatigue scores in type 2 diabetic patients, albeit this variable correlated with both inflammation and specific fatigue dimensions in those patients and while coefficient regressions decreased somewhat when adiposity was controlled for in regression models. This finding highlights the possibility that the few associations that were measured between BMI and fatigue scores in type 2 diabetic patients were due to the interrelationship of adiposity with inflammatory markers. Moreover, this result suggests that, while related to adiposity, systemic inflammation has a specific and independent/direct effect (unmediated by adiposity characteristics) on fatigue symptoms in type 2 diabetes. Among the biological markers that were assessed in this study, hsIL-6 and hsCRP were those showing the strongest connections with fatigue scores, notably with the dimensions of mental fatigue, reduced motivation and reduced activity, in type 2 diabetic patients. This finding is consistent with previous reports (some of them emanating from our group) indicating the involvement of IL-6 and/or CRP in fatigue symptoms and neurobehavioral alterations in patients afflicted with chronic conditions (e.g., metabolic disorders, chronic fatigue syndrome, cancers) (Bower et al., 2011; Capuron et al., 2008, 2011b; Collado-Hidalgo et al., 2006;
Fletcher et al., 2009; Orre et al., 2009, 2011) and in healthy models of chronic low grade inflammation such as normal aging (Capuron et al., 2011a; Valentine et al., 2011). Similar associations have been reported in healthy individuals (Cho et al., 2009; Rief et al., 2010; Thomas et al., 2011). Interestingly, higher neopterin concentrations were also found to relate to increased scores of general fatigue in the type 2 diabetic population from the present study. Surprisingly, albeit type 2 diabetic patients exhibited high scores of physical fatigue, no significant relationship was found between physical fatigue and levels of inflammatory markers. This result may be explained by the relatively small samples of diabetic subjects recruited in the present study. This limitation may have lead to reductions in statistical power, notably in linear regression models that controlled for multiple covariates with potential confounding effect. This limitation warrants further investigations on larger diabetic populations to comfort findings from the present study.

The mechanisms by which systemic inflammation may participate in the physiopathology of fatigue symptoms in patients with type 2 diabetes remain to be elucidated. Such mechanisms may include specific interactions between metabolic and inflammatory processes leading to subsequent alterations in brain chemistry (e.g., neurotransmitter and endocrine systems) and neurocircuitry involving notably the basal ganglia and fronto-thalamic loops (Capuron and Miller, 2011; Majer et al., 2008; Rocca et al., 2007). In addition, enzymatic variations responsible for alterations in the metabolism of neurotransmitters may be involved as inflammatory factors have potent effects of the activity of the enzymes, indoleamine-2,3-dioxygenase (IDO) and GTP cyclohydrolase-1 (GTP-CH1), involved in the biosynthesis of noradrenalin, dopamine and serotonin. Further clinical and experimental studies are needed to specifically address this question.

One question remains regarding the possibilities to prevent and/or treat diabetes-related fatigue - and subsequently improve patients' quality of life - using interventions to reduce inflammation. The association measured in the present study between adiposity and inflammatory markers supports the idea that reduced inflammation may be achieved by weight loss strategies, as shown in previous reports (Viardot et al., 2010). Nutritional strategies may be also envisaged to modulate inflammation and its central/behavioral effects in patients with type 2 diabetes. Omega- 3 polyunsaturated fatty acids ( $n-3$ PUFA) may represent good candidates for such a strategy, as these nutrients have been shown to exert potent anti-inflammatory effects (Calder, 2006). Relevant to diabetes, $n-3$ PUFA supplements were found to downregulate inflammation-related genes in the adipose tissue of women with type 2 diabetes (Kabir et al., 2007). In addition, these nutrients have been shown to reduce or prevent insulin resistance in experimental and clinical studies, although results in clinical settings are less consistent than in animal studies (Poudyal et al., 2011 review; Storlien et al., 1987). Finally, exercise training may represent another alternative since this strategy was found to reduce inflammatory markers in overweight individuals with type 2 diabetes (Kadoglou et al., 2007).

In conclusion, the present findings indicate that systemic lowgrade inflammation is associated with fatigue symptom dimensions in patients with type 2 diabetes and suggest the involvement of inflammatory processes in the pathophysiology of diabetes-related fatigue.

\section{Funding}

This study was supported by subventions from the INRA, Region Aquitaine, and by the European Community (6th framework program) (Grant No. IRG2006-039575, LC). 
Table 5

Relationship of inflammatory markers with fatigue symptoms in patients with type 2 diabetes.

\begin{tabular}{|c|c|c|c|c|c|c|c|c|c|c|c|c|c|}
\hline \multirow[t]{2}{*}{ Fatigue symptoms } & \multirow{2}{*}{$\begin{array}{l}\text { Predictors } \\
\text { Inflammatory markers }\end{array}$} & \multicolumn{3}{|c|}{ Model 1} & \multicolumn{3}{|c|}{ Model 2 (adjusting for BMI) } & \multicolumn{2}{|c|}{$\underline{\text { Mediation Sobel }}$} & & \multicolumn{3}{|c|}{$\underline{\text { Separate effects of covariates }}$} \\
\hline & & $\beta$ & $F$ & $p$ & $\beta$ & $F$ & $p$ & $Z$ & $p$ & & $\beta$ & $F$ & $p$ \\
\hline \multirow[t]{5}{*}{ Total fatigue } & hsCRP & .755 & 5.4 & $<.05$ & .553 & 2.0 & .18 & .88 & .38 & Diabetes duration & .242 & .9 & .35 \\
\hline & hsIL6 & .650 & 11.6 & $<.01$ & .677 & 5.3 & $<.05$ & -.12 & .90 & HbA1C levels & .025 & .01 & .92 \\
\hline & hsTNF- $-\alpha$ & .059 & .04 & .84 & -.217 & .6 & .46 & & & Insulin treatment & .322 & 1.7 & .21 \\
\hline & Neopterin & .451 & 2.3 & .16 & .305 & 1.1 & .32 & & & Fasting status & .293 & 1.4 & .25 \\
\hline & Composite inflammation & .610 & 6.7 & $<.05$ & .499 & 2.2 & .17 & .47 & .64 & & & & \\
\hline \multirow[t]{5}{*}{ General fatigue } & hsCRP & .222 & .6 & .45 & .177 & .2 & .63 & & & Diabetes duration & .496 & 4.9 & $<.05$ \\
\hline & hsIL6 & .256 & 1.9 & .19 & .362 & 1.6 & .23 & & & HbA1C levels & -.091 & .1 & .73 \\
\hline & hsTNF- $\alpha$ & .042 & .04 & .85 & -.024 & .01 & .92 & & & Insulin treatment & .503 & 5.1 & $<.05$ \\
\hline & Neopterin & .471 & 6.0 & $<.05$ & .473 & 4.9 & $<.05$ & -.03 & .98 & Fasting status & .520 & 5.6 & $<.05$ \\
\hline & Composite inflammation & .304 & 2.3 & .16 & .405 & 2.0 & .19 & & & & & & \\
\hline \multirow[t]{5}{*}{ Reduced activity } & hsCRP & .698 & 3.5 & .09 & .748 & 2.6 & .13 & -.20 & .84 & Diabetes duration & .085 & .1 & .75 \\
\hline & hsIL6 & .483 & 3.6 & .08 & .711 & 3.5 & .09 & -.79 & .43 & HbA1C levels & .151 & .3 & .56 \\
\hline & hsTNF- $\alpha$ & -.150 & .2 & .63 & -.329 & .9 & .35 & & & Insulin treatment & .108 & .2 & .68 \\
\hline & Neopterin & .052 & .02 & .88 & -.034 & .01 & .93 & & & Fasting status & .059 & .05 & .82 \\
\hline & Composite inflammation & .360 & 1.4 & .26 & .378 & .7 & .41 & & & & & & \\
\hline \multirow[t]{5}{*}{ Mental fatigue } & hsCRP & .903 & 8.8 & $<.05$ & .623 & 3.3 & .09 & 1.23 & .22 & Diabetes duration & .009 & .01 & .97 \\
\hline & hsIL6 & .707 & 14.9 & $<.01$ & .600 & 4.6 & .05 & .52 & .61 & HbA1C levels & -.075 & .1 & .78 \\
\hline & hsTNF- $\alpha$ & .155 & .3 & .60 & -.172 & .4 & .53 & & & Insulin treatment & .375 & 2.4 & .14 \\
\hline & Neopterin & .408 & 1.7 & .22 & .204 & .5 & .48 & & & Fasting status & .295 & 1.4 & .25 \\
\hline & Composite inflammation & .689 & 9.2 & $<.05$ & .468 & 2.2 & .17 & .97 & .33 & & & & \\
\hline \multirow[t]{5}{*}{ Reduced motivation } & hsCRP & .930 & 9.1 & $<.05$ & .663 & 3.6 & .09 & 1.17 & .24 & Diabetes duration & -.077 & .1 & .77 \\
\hline & hsIL6 & .765 & 20.2 & .001 & .728 & 7.7 & $<.05$ & .19 & .85 & HbA1C levels & .252 & 1.0 & .33 \\
\hline & hsTNF $-\alpha$ & .225 & .6 & .45 & -.081 & .09 & .78 & & & Insulin treatment & .074 & .1 & .78 \\
\hline & Neopterin & .427 & 1.8 & .20 & .225 & .6 & .46 & & & Fasting status & -.029 & .01 & .91 \\
\hline & Composite inflammation & .745 & 11.5 & $<.01$ & .577 & 3.5 & .09 & .76 & .45 & & & & \\
\hline
\end{tabular}

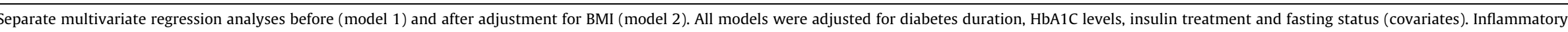

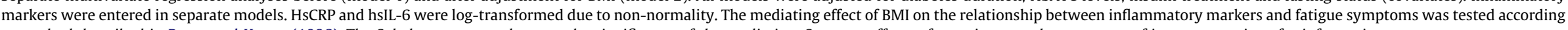
to method described in Baron and Kenny (1986). The Sobel test was used to test the significance of the mediation. Separate effects of covariates on the outcomes of interest are given for information.

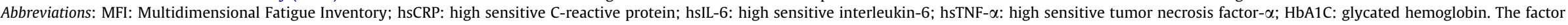

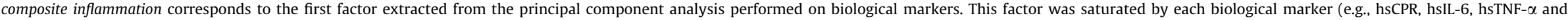
neopterin) and explained $49 \%$ of the variance. 


\section{References}

Alexandraki, K.I., Piperi, C., Ziakas, P.D., Apostolopoulos, N.V., Makrilakis, K., Syriou, V., Diamanti-Kandarakis, E., Kaltsas, G., Kalofoutis, A., 2008. Cytokine secretion in long-standing diabetes mellitus type 1 and 2: associations with low-grade systemic inflammation. J. Clin. Immunol. 28, 314-321.

Baris, N., Erdogan, M., Sezer, E., Saygili, F., Mert Ozgonul, A., Turgan, N., Ersoz, B., 2009. Alterations in L-arginine and inflammatory markers in type 2 diabetic patients with and without microalbuminuria. Acta Diabetol. 46, 309-316.

Baron, R.M., Kenny, D.A., 1986. The moderator-mediator variable distinction in social psychological research: conceptual, strategic, and statistical considerations. J. Pers. Soc. Psychol. 51, 1173-1182.

Basu, S., Larsson, A., Vessby, J., Vessby, B., Berne, C., 2005. Type 1 diabetes is associated with increased cyclooxygenase- and cytokine-mediated inflammation. Diabetes Care 28, 1371-1375.

Bower, J.E., 2007. Cancer-related fatigue: links with inflammation in cancer patients and survivors. Brain Behav. Immun. 21, 863-871.

Bower, J.E., Ganz, P.A., Aziz, N., Fahey, J.L., 2002. Fatigue and proinflammatory cytokine activity in breast cancer survivors. Psychosom. Med. 64, 604-611.

Bower, J.E., Ganz, P.A., Irwin, M.R., Kwan, L., Breen, E.C., Cole, S.W., 2011. Inflammation and behavioral symptoms after breast cancer treatment: do fatigue, depression, and sleep disturbance share a common underlying mechanism? J. Clin. Oncol. 29, 3517-3522.

Calder, P.C., 2006. N-3 polyunsaturated fatty acids, inflammation, and inflammatory diseases. Am. J. Clin. Nutr. 83, 1505S-1519S.

Capuron, L., Gumnick, J.F., Musselman, D.L., Lawson, D.H., Reemsnyder, A., Nemeroff, C.B., Miller, A.H., 2002. Neurobehavioral effects of interferon-alpha in cancer patients: phenomenology and paroxetine responsiveness of symptom dimensions. Neuropsychopharmacology 26, 643-652.

Capuron, L., Pagnoni, G., Demetrashvili, M.F., Lawson, D.H., Fornwalt, F.B., Woolwine, B., Berns, G.S., Nemeroff, C.B., Miller, A.H., 2007. Basal ganglia hypermetabolism and symptoms of fatigue during interferon-alpha therapy. Neuropsychopharmacology 32, 2384-2392.

Capuron, L., Su, S., Miller, A.H., Bremner, J.D., Goldberg, J., Vogt, G.J., Maisano, C., Jones, L., Murrah, N.V., Vaccarino, V., 2008. Depressive symptoms and metabolic syndrome: is inflammation the underlying link? Biol. Psychiatry 64, 896-900.

Capuron, L., Schroecksnadel, S., Feart, C., Aubert, A., Higueret, D., Barberger-Gateau, P., Laye, S., Fuchs, D., 2011a. Chronic low-grade inflammation in elderly persons is associated with altered tryptophan and tyrosine metabolism: role in neuropsychiatric symptoms. Biol. Psychiatry 70, 175-182.

Capuron, L., Poitou, C., Machaux-Tholliez, D., Frochot, V., Bouillot, J.L., Basdevant, A., Layé, S., Clément, K., 2011b. Relationship between adiposity, emotional status and eating behaviour in obese women: role of inflammation. Psychol. Med. 41, 1517-1528.

Capuron, L., Miller, A.H., 2011. Immune system to brain signaling: neuropsychopharmacological implications. Pharmacol. Ther. 130, 226-238.

Cavelti-Weder, C., Furrer, R., Keller, C., Babians-Brunner, A., Solinger, A.M., Gast, H., Fontana, A., Donath, M.Y., Penner, I.K., 2011. Inhibition of IL-1beta improves fatigue in type 2 diabetes. Diabetes Care 34, e158.

Cho, H.J., Seeman, T.E., Bower, J.E., Kiefe, C.I., Irwin, M.R., 2009. Prospective association between C-reactive protein and fatigue in the coronary artery risk development in young adults study. Biol. Psychiatry 66, 871-878.

Collado-Hidalgo, A., Bower, J.E., Ganz, P.A., Cole, S.W., Irwin, M.R., 2006. Inflammatory biomarkers for persistent fatigue in breast cancer survivors. Clin. Cancer Res. 12, 2759-2766.

Dantzer, R., O’Connor, J.C., Freund, G.G., Johnson, R.W., Kelley, K.W., 2008. From inflammation to sickness and depression: when the immune system subjugates the brain. Nat. Rev. Neurosci. 9, 46-56.

Devaraj, S., Glaser, N., Griffen, S., Wang-Polagruto, J., Miguelino, E., Jialal, I., 2006. Increased monocytic activity and biomarkers of inflammation in patients with type 1 diabetes. Diabetes 55, 774-779.

Devaraj, S., Cheung, A.T., Jialal, I., Griffen, S.C., Nguyen, D., Glaser, N., Aoki, T., 2007. Evidence of increased inflammation and microcirculatory abnormalities in patients with type 1 diabetes and their role in microvascular complications. Diabetes 56, 2790-2796.

Drivsholm, T., de Fine Olivarius, N., Nielsen, A.B., Siersma, V., 2005. Symptoms, signs and complications in newly diagnosed type 2 diabetic patients, and their relationship to glycaemia, blood pressure and weight. Diabetologia 48, 210214

Duncan, B.B., Schmidt, M.I., Pankow, J.S., Ballantyne, C.M., Couper, D., Vigo, A., Hoogeveen, R., Folsom, A.R., Heiss, G., 2003. Low-grade systemic inflammation and the development of type 2 diabetes: the atherosclerosis risk in communities study. Diabetes 52, 1799-1805.

Festa, A., D'Agostino Jr., R., Tracy, R.P., Haffner, S.M., 2002. Elevated levels of acutephase proteins and plasminogen activator inhibitor-1 predict the development of type 2 diabetes: the insulin resistance atherosclerosis study. Diabetes 51, 1131-1137.

Flachenecker, P., Bihler, I., Weber, F., Gottschalk, M., Toyka, K.V., Rieckmann, P., 2004. Cytokine mRNA expression in patients with multiple sclerosis and fatigue. Mult. Scler. 10, 165-169.

Fletcher, M.A., Zeng, X.R., Barnes, Z., Levis, S., Klimas, N.G., 2009. Plasma cytokines in women with chronic fatigue syndrome. J. Transl. Med. 7, 96.

Fritschi, C., Quinn, L., 2010. Fatigue in patients with diabetes: a review. J. Psychosom. Res. 69, 33-41.
Heesen, C., Nawrath, L., Reich, C., Bauer, N., Schulz, K.H., Gold, S.M., 2006. Fatigue in multiple sclerosis: an example of cytokine mediated sickness behaviour? J. Neurol. Neurosurg. Psychiatry 77, 34-39.

Hu, F.B., Meigs, J.B., Li, T.Y., Rifai, N., Manson, J.E., 2004. Inflammatory markers and risk of developing type 2 diabetes in women. Diabetes 53, 693-700.

Kabir, M., Skurnik, G., Naour, N., Pechtner, V., Meugnier, E., Rome, S., QuignardBoulange, A., Vidal, H., Slama, G., Clement, K., Guerre-Millo, M., Rizkalla, S.W. 2007. Treatment for 2 mo with $\mathrm{n} 3$ polyunsaturated fatty acids reduces adiposity and some atherogenic factors but does not improve insulin sensitivity in women with type 2 diabetes: a randomized controlled study. Am. J. Clin. Nutr. 86, 1670-1679.

Kadoglou, N.P., Perrea, D., Iliadis, F., Angelopoulou, N., Liapis, C., Alevizos, M., 2007. Exercise reduces resistin and inflammatory cytokines in patients with type 2 diabetes. Diabetes Care 30, 719-721.

Kelley, K.W., Bluthe, R.M., Dantzer, R., Zhou, J.H., Shen, W.H., Johnson, R.W. Broussard, S.R., 2003. Cytokine-induced sickness behavior. Brain Behav. Immun. 17 (Suppl. 1), S112-118.

Lasselin, J., Layé, S., Barreau, J.B., Rivet, A., Dulucq, M.J., Gin, H., Capuron, L., 2012 Fatigue and cognitive symptoms in patients with diabetes: relationship with disease phenotype and insulin treatment. Psychoneuroendocrinology. http:// dx.doi.org/10.1016/j.psyneuen.2012.01.016.

Majer, M., Welberg, L.A., Capuron, L., Pagnoni, G., Raison, C.L., Miller, A.H., 2008. IFNalpha-induced motor slowing is associated with increased depression and fatigue in patients with chronic hepatitis C. Brain Behav. Immun. 22, 870-880.

Miller, A.H., Ancoli-Israel, S., Bower, J.E., Capuron, L., Irwin, M.R., 2008. Neuroendocrine-immune mechanisms of behavioral comorbidities in patients with cancer. J. Clin. Oncol. 26, 971-982.

Mraz, M., Lacinova, Z., Drapalova, J., Haluzikova, D., Horinek, A., Matoulek, M., Trachta, P., Kavalkova, P., Svacina, S., Haluzik, M., 2011. The effect of very-lowcalorie diet on mRNA expression of inflammation-related genes in subcutaneous adipose tissue and peripheral monocytes of obese patients with type 2 diabetes mellitus. J. Clin. Endocrinol. Metab. 96, E606-E613.

Orre, I.J., Murison, R., Dahl, A.A., Ueland, T., Aukrust, P., Fossa, S.D., 2009. Levels of circulating interleukin-1 receptor antagonist and C-reactive protein in longterm survivors of testicular cancer with chronic cancer-related fatigue. Brain Behav. Immun. 23, 868-874.

Orre, I.J., Reinertsen, K.V., Aukrust, P., Dahl, A.A., Fossa, S.D., Ueland, T., Murison, R. 2011. Higher levels of fatigue are associated with higher CRP levels in diseasefree breast cancer survivors. J. Psychosom. Res. 71, 136-141.

Pedersen, M., Bruunsgaard, H., Weis, N., Hendel, H.W., Andreassen, B.U., Eldrup, E. Dela, F., Pedersen, B.K., 2003. Circulating levels of TNF-alpha and IL-6-relation to truncal fat mass and muscle mass in healthy elderly individuals and in patients with type-2 diabetes. Mech. Ageing Dev. 124, 495-502.

Pham, M.N., Hawa, M.I., Pfleger, C., Roden, M., Schernthaner, G., Pozzilli, P. Buzzetti, R., Scherbaum, W., Seissler, J., Kolb, H., Hunter, S., Leslie, R.D., Schloot, N.C., 2011. Pro- and anti-inflammatory cytokines in latent autoimmune diabetes in adults, type 1 and type 2 diabetes patients: action LADA 4. Diabetologia 54 1630-1638.

Pickup, J.C., Chusney, G.D., Thomas, S.M., Burt, D., 2000. Plasma interleukin-6 tumour necrosis factor alpha and blood cytokine production in type 2 diabetes. Life Sci. 67, 291-300.

Poudyal, H., Panchal, S.K., Diwan, V., Brown, L., 2011. Omega-3 fatty acids and metabolic syndrome: effects and emerging mechanisms of action. Prog. Lipid Res. 50, 372-387.

Preacher, K.J., Hayes, A.F., 2008. Asymptotic and resampling strategies for assessing and comparing indirect effects in multiple mediator models. Behav. Res. Methods 40, 879-891.

Raison, C.L., Lin, J.M., Reeves, W.C., 2009. Association of peripheral inflammatory markers with chronic fatigue in a population-based sample. Brain Behav. Immun. 23, 327-337.

Rief, W., Mills, P.J., Ancoli-Israel, S., Ziegler, M.G., Pung, M.A., Dimsdale, J.E., 2010 Overnight changes of immune parameters and catecholamines are associated with mood and stress. Psychosom. Med. 72, 755-762.

Rocca, M.A., Agosta, F., Colombo, B., Mezzapesa, D.M., Falini, A., Comi, G., Filippi, M. 2007. FMRI changes in relapsing-remitting multiple sclerosis patients complaining of fatigue after IFNbeta-1 a injection. Hum. Brain Mapp. 28, 373-382.

Sam, S., Haffner, S., Davidson, M.H., D’Agostino Sr., R.B., Feinstein, S., Kondos, G., Perez, A., Mazzone, T., 2009. Relation of abdominal fat depots to systemic markers of inflammation in type 2 diabetes. Diabetes Care 32, 932-937.

Schmidt, M.I., Duncan, B.B., Sharrett, A.R., Lindberg, G., Savage, P.J., Offenbacher, S., Azambuja, M.I., Tracy, R.P., Heiss, G., 1999. Markers of inflammation and prediction of diabetes mellitus in adults (Atherosclerosis Risk in Communities study): a cohort study. Lancet 353, 1649-1652.

Sheehan, D.V., Lecrubier, Y., Sheehan, K.H., Amorim, P., Janavs, J., Weiller, E., Hergueta, T., Baker, R., Dunbar, G.C., 1998. The Mini-International Neuropsychiatric Interview (M.I.N.I.): the development and validation of a structured diagnostic psychiatric interview for DSM-IV and ICD-10. J. Clin. Psychiatry 59(Suppl. 20, 22-33), quiz 34-57.

Smets, E.M., Garssen, B., Bonke, B., De Haes, J.C., 1995. The Multidimensional Fatigue Inventory (MFI) psychometric qualities of an instrument to assess fatigue. J. Psychosom. Res. 39, 315-325.

Spranger, J., Kroke, A., Mohlig, M., Hoffmann, K., Bergmann, M.M., Ristow, M. Boeing, H., Pfeiffer, A.F., 2003. Inflammatory cytokines and the risk to develop type 2 diabetes: results of the prospective population-based European 
Prospective Investigation into Cancer and Nutrition (EPIC)-Potsdam Study. Diabetes 52, 812-817.

Storlien, L.H., Kraegen, E.W., Chisholm, D.J., Ford, G.L., Bruce, D.G., Pascoe, W.S. 1987. Fish oil prevents insulin resistance induced by high-fat feeding in rats. Science 237, 885-888.

Su, S.C., Pei, D., Hsieh, C.H., Hsiao, F.C., Wu, C.Z., Hung, Y.J., 2011. Circulating proinflammatory cytokines and adiponectin in young men with type 2 diabetes. Acta Diabetol. 48, 113-119.

Thomas, K.S., Motivala, S., Olmstead, R., Irwin, M.R., 2011. Sleep depth and fatigue: role of cellular inflammatory activation. Brain Behav. Immun. 25, 53-58.

Trayhurn, P., Wood, I.S., 2004. Adipokines: inflammation and the pleiotropic role of white adipose tissue. Br. J. Nutr. 92, 347-355.
Treszl, A., Szereday, L., Doria, A., King, G.L., Orban, T., 2004. Elevated C-reactive protein levels do not correspond to autoimmunity in type 1 diabetes. Diabetes Care 27, 2769-2770.

Valentine, R.J., Woods, J.A., McAuley, E., Dantzer, R., Evans, E.M., 2011. The associations of adiposity, physical activity and inflammation with fatigue in older adults. Brain Behav. Immun. 25, 1482-1490.

Viardot, A., Lord, R.V., Samaras, K., 2010. The effects of weight loss and gastric banding on the innate and adaptive immune system in type 2 diabetes and prediabetes. J. Clin. Endocrinol. Metab. 95, 2845-2850.

Wellen, K.E., Hotamisligil, G.S., 2003. Obesity-induced inflammatory changes in adipose tissue. J. Clin. Invest. 112, 1785-1788. 